Dermatology 2009;219:179-181

DOI: $10.1159 / 000228319$

\section{Red Scalp Disease - A Rosacea-Like Dermatosis of the Scalp? Successful Therapy with Oral Tetracycline}

Patrick A. Oberholzer, Stephan Nobbe, Isabel Kolm, Katrin Kerl, Jivko Kamarachev, Ralph M. Trüeb

Department of Dermatology, University Hospital Zurich, Zurich, Switzerland

Key Words

Red scalp disease $\cdot$ Rosacea-like dermatosis of the scalp • Tetracycline therapy

Red scalp disease is a common complaint and therapeutic challenge encountered in daily clinical practice. We report 2 cases of red scalp disease with clinical and histopathologic findings consistent with rosacea and response to oral tetracycline therapy. The discussion includes a review of the literature on red scalp disease as well as our interpretation of the entity, particularly a possible link to rosacea.

\section{Case Reports}

A 57-year-old male Caucasian was referred by his general practitioner with a 3-year history of a red and itchy scalp (fig. 1a). He had a medical record of diabetes mellitus type 2, arterial hypertension, renal insufficiency, chronic lumbar pain, osteoarthritis of the hip, alcohol abuse and cigarette smoking. His regular medication included insulin, aspirin, atenolol, tamsulosin, darbepoetin $\alpha$, iron sucrose injections, oxazepam, biotin and folic acid. No allergies were annotated. The situation had worsenend during the last 6 months. Previous treatments included oral antihistamines, diverse medicated antiseborrheic shampoos and topical corticosteroid applications without success. Clinical examination of the scalp, including dermoscopy, revealed multifocal, telangiectatic erythema with follicular papules and few pustules, without necrosis (fig. 1b). The lesions were limited to the hairy scalp. There were no associated skin lesions elsewhere. Microbiologic studies did not reveal any microbial pathogens.

A 57-year-old Caucasian female presented with the complaint of burning sensations of the scalp. She had been treated before by her private dermatologist with antiseborrheic shampoos, again without success. Since her complaints continued, she seeked advice. Otherwise she was well, with no medical record history of significance, no allergies and no medication on a regular basis. Clinical examination again revealed a red scalp with follicular erythematous, telangiectatic papules, strictly within the limits of her scalp hair (fig. $2 \mathrm{a}-\mathrm{c}$ ). A bacterial swab revealed normal skin flora.

Both patients presented with the complaint of a red scalp with itching or burning sensations and a very similar clinical presentation of erythema, telangiectasia and follicular papules and pustules of the scalp. Lesional biopsies taken from both patients showed telangiectasia, perifollicular mixed-cellular inflammation with granuloma formation (fig. 1c) in the mid dermis consistent with rosacea and moderate elastosis. No fungal spores were found. The direct immunofluorescence of both patients was negative.

On the basis of clinical and histopathologic features of rosacea, a therapeutic trial with oral tetracyclines was done. After 6 weeks of oral lymecycline $(300 \mathrm{mg} /$ day $)$, the patients reported significant improvement of their complaints. On clinical examination, erythema and follicular papules and pustules had disappeared; there remained residual telangiectasia (fig. 2d, e). Patients were also recommended to omit topical corticosteroids and to protect their scalp from UV exposure.

\section{Discussion}

Red scalp disease was originally reported by Thestrup-Pedersen and Hjorth [1] in 1987 and subsequently commented on by Moschella [2] in 1994 who described the difficult problem of 'diffuse red scalp disease which can also be itchy and burning, is nonresponsive to any therapy including potent topical steroids or antiseborrhoeic therapy'. More recently, Ramon Grimalt presented findings in 18 patients with 'red scalp syndrome' at the $2000 \mathrm{An}$ nual Meeting of the European Hair Research Society. By definition, no specific dermatologic disease typically affecting the scalp, such as eczema or psoriasis, was found. The differential diagnosis includes contact dermatitis (irritative and allergic), seborrheic dermatitis, psoriasis, lichen planopilaris, lupus erythematosus and dermatomyositis. Acne miliaris necrotica may also present with itchy follicular lesions, though with a tendency to necrosis and without the diffuse erythema typical of red scalp disease. In the 18 patients with red scalp presented by Grimalt, scalp redness was associated with hair loss due to androgenetic alopecia in the majority of cases, leading to speculations over the role of chronic UV exposure of the balding scalp [3]. Moreover, dilated and tortuous vessels are typically found in photodamaged skin. Other pathogenetic speculations have concerned perifollicular inflammation and increased expression of the neuropeptide substance $\mathrm{P}$ in the vicinity of affected hair follicles. These findings suggest a connection between sensory or subjective irritation and cutaneous vascular reactivity. Willimann and Trüeb [4] drew attention to a correlation of telangiectasia of the scalp with the presence of scalp discomfort, and proposed an analogy to the observation of Lonne-Rahm et al. [5], who found that patients with a telangiectactic type of rosacea respond more frequently with stinging sen-

\section{KARGER}

(c) 2009 S. Karger AG, Basel

Fax +4161306 1234

E-Mail karger@karger.ch

www.karger.com
Accessible online at: www.karger.com/drm 

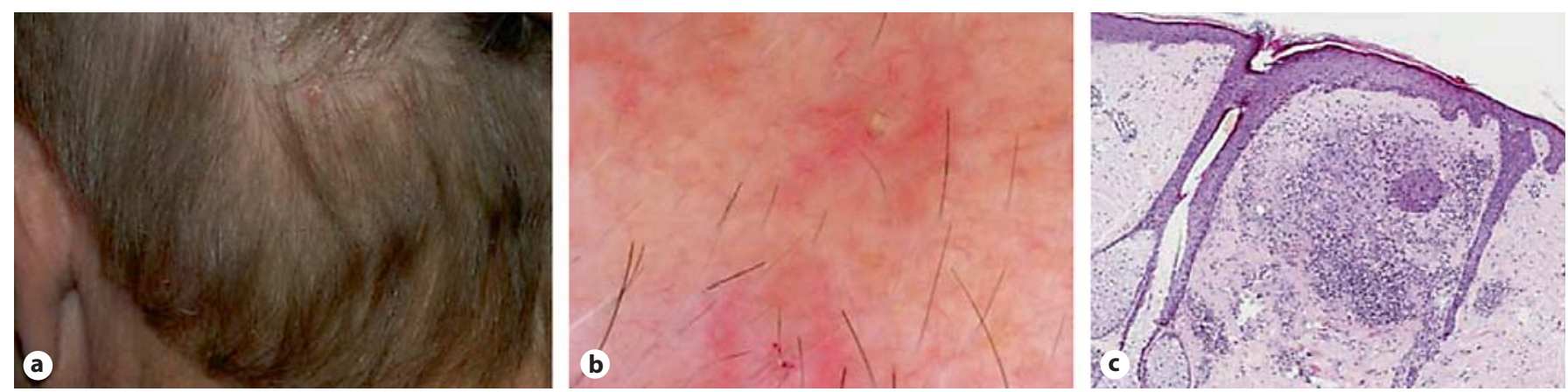

Fig. 1. Rosacea of the scalp (patient 1). a Clinical presentation. b Dermoscopic finding. c Histology. HE.
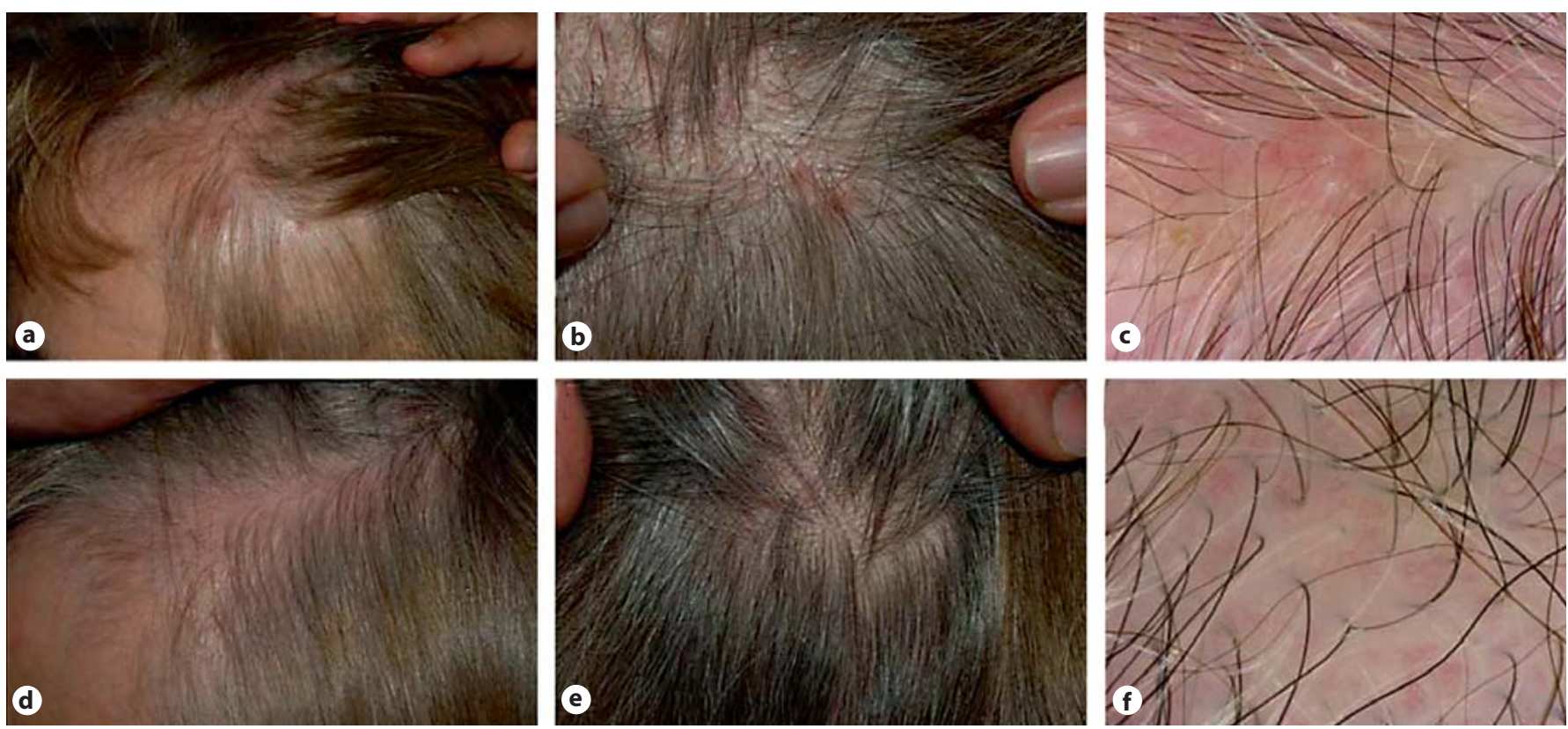

Fig. 2. Clinical and dermoscopic findings before (a-c) and after (d-f) 6 weeks of treatment with lymecycline (patient 2).

sations to the topical application of $5 \%$ lactic acid on the cheeks than patients with the papulopustular type of rosacea or normal controls.

On the basis of our observation of clinical and histopathologic features of rosacea in our patients, and the response to oral tetracycline therapy, we suggest that patients complaining of red scalp accompanied with scalp discomfort may present a rosacea-like dermatosis of the scalp. Careful examination of the affected scalp, including dermoscopy, invariably reveals erythema, telangiectasia, follicular papules and pustules. Histology demonstrates ectatic venules and a usually sparse perivascular infiltrate, as well as in the case of papules a more pleomorphic perifollicular infiltration with granuloma formation, including neutrophils. Intrafollicular collections of neutrophils are found when pustules are observed.
Rosacea is a chronic inflammatory disorder usually affecting the central parts of the face with flushing, persistent erythema and telangiectasia, in combination with episodes of swelling, papules and pustules. It typically presents either as erythematotelangiectatic rosacea or as the papulopustular type [6]. Eye involvement (ocular rosacea) is common, and other extrafacial locations may be involved more frequently than appreciated because of failure to search [7-11]; in particular, rosacea affecting the upper forehead and bald scalp has been reported [12-14]. To the best of our knowledge, as yet no report affecting the hairy scalp has been published. For this reason, it seems prudent to preliminarily refer to our observation as rosacea-like dermatosis of the scalp before definitely interpreting it as yet another form of extrafacial rosacea - namely of the hairy scalp. 


\section{References}

$\checkmark 1$ Thestrup-Pedersen K, Hjorth N: Red scalp: a previously undescribed disease of the scalp? Ugeskr Laeger 1987;149:2141-2142.

2 Bernhard JD: Itch: Mechanisms and Management of Pruritus. New York, McGraw-Hill, 1994, p 51.

3 Trüeb RM: Is androgenetic alopecia a photoaggravated dermatosis? Dermatology 2003;207:343-348.

4 Willimann B, Trüeb RM: Hair pain (trichodynia): frequency and relationship to hair loss and patient gender. Dermatology 2002;205:374377.

5 Lonne-Rahm SB, Fischer T, Berg M: Stinging and rosacea. Acta Derm Venereol 1999;79:460-461.

6 Wilkin J, Dahl M, Detmar M, Drake L, Feinstein A, Odom R, Powell F: Standard classification of rosacea: report of the national rosacea society expert committee on the classification and staging of rosacea. J Am Acad Dermatol 2002;46:584-587.

7 Ayres S Jr: Extrafacial rosacea is rare but does exist. J Am Acad Dermatol 1987;16:391-392.

$>8$ Dupont C: How common is extrafacial rosacea? J Am Acad Dermatol 1986; 14:839.
9 Marks R, Jones EW: Disseminated rosacea. Br J Dermatol 1969;81:1628.

10 Pereira TM, Vieira AP, Basto AS: Rosacea with extensive extrafacial lesions. Int J Dermatol 2008;47:52-55.

11 Wilkin JK: Epigastric rosacea. Arch Dermatol 1980;116:584.

12 Gajewska M: Rosacea of common male baldness. Br J Dermatol 1975; 93:63-66.

13 Miskjian HG: Demodicidosis (demodex infestation of the scalp). AMA Arch Derm Syphilol 1951;63:282-283.

14 Wong CS, Kirby B: Demodicidosis in scalp rosacea? Clin Exp Dermatol 2004;29:318-319.

Prof. Ralph M. Trüeb

Department of Dermatology, Gloriastrasse 31

$\mathrm{CH}-8091$ Zürich (Switzerland)

Tel. +41 4425530 79, Fax +41 442554431

E-Mail ralph.trueeb@usz.ch 